\title{
Universal Chaotic Scattering on Quantum Graphs
}

\author{
Z. Pluhař $\check{r}^{a}$ and H. A. Weidenmüller ${ }^{2 *}$ \\ ${ }^{a}$ Faculty of Mathematics and Physics, Charles University, 18000 Praha 8, Czech Republic \\ ${ }^{b}$ Max-Planck-Institut für Kernphysik, 69029 Heidelberg, Germany
}

\begin{abstract}
We calculate the $S$-matrix correlation function for chaotic scattering on quantum graphs and show that it agrees with that of random-matrix theory (RMT). We also calculate all higher $S$-matrix correlation functions in the Ericson regime. These, too, agree with RMT results as far as the latter are known. We conjecture that our results give a universal description of chaotic scattering.
\end{abstract}

PACS numbers: 05.45.Mt, 03.65.Nk, 24.60.Dr

Purpose. Closed quantum systems that are chaotic in the classical limit possess universal spectral fluctuation properties. Depending on symmetry, these coincide with the fluctuation properties of one of Dyson's three canonical random-matrix ensembles [1]. These statements, originally formulated in the form of a conjecture [2], have since been demonstrated for the two-point level correlator of general chaotic systems [3, 4] and of chaotic quantum graphs [5].

For open chaotic quantum systems, the fluctuation properties of the scattering matrix ( $S$-matrix) are at issue, quantified in terms of the totality of $S$-matrix correlation functions. As for closed systems, it would be desirable to establish (at least) the complete equivalence between the $S$-matrix two-point correlation function for chaotic scattering with that of random-matrix theory (RMT) given in Ref. [6] for the orthogonal and in Ref. 7] for the unitary case. However, we are not aware of any analytical results for $S$-matrix correlations for chaotic scattering.

In the present Letter, we start to fill that gap. For the case of orthogonal symmetry, we calculate the $S_{-}$ matrix two-point correlation function analytically for open chaotic quantum graphs. We show that our result coincides with that 6] of RMT. We also calculate all higher $S$-matrix correlation functions in the Ericson regime. These coincide with RMT results as far as the latter are known $[6,[8,[9]$. In that regime, the $S$-matrix elements are supposed [10, 11] to have a Gaussian distribution. Our results show that this is the case only for strong absorption in all channels. We conjecture that our results are universal, i.e., apply to chaotic scattering in general.

We focus attention on chaotic quantum graphs because here the semiclassical expansion is exact and scattering theory is particularly transparent. Chaotic scattering on quantum graphs was introduced in Refs. [12, 13] where many of its properties were displayed with the help of numerical simulations, see also Refs. [14].

Scattering Matrix. Our presentation is self-contained but largely follows the developments of Ref. [13]. A graph

*Electronic address: Hans.Weidenmueller@mpi-hd.mpg.de is a system of $V$ vertices labelled $\alpha, \beta, \ldots$ that are linked by $B$ bonds. For simplicity of notation we assume that every vertex $\alpha$ is linked by a single bond $(\alpha \beta)$ to every other vertex $\beta$ ("completely connected graph"). Then the number of bonds is $B=V(V-1) / 2$. Our results remain valid, however, if some bonds are missing, see the discussion under "massive modes" below. The lengths $L_{b}$ of all bonds $b=(\alpha \beta)$ are assumed to be similar (so that $L_{\min } \leq L_{b} \leq L_{\max }$ for all $b$ ) and incommensurate. That assumption is neccessary for the graph to be chaotic. A number $\Lambda \geq 1$ of vertices is linked by a single bond each (a "lead") to infinity. The number $\Lambda$ of leads defines both, the number of scattering channels and the dimension of the $S$-matrix. In analogy to the RMT approach (where the dimension $N$ of the Hamiltonian matrix is taken to infinity while the number of channels is kept fixed) we let $V \rightarrow \infty$ but keep $\Lambda$ fixed.

On each bond or lead, waves propagate freely with wave number $k$ (the same for all bonds/leads), and the wave function is a linear combination of amplitudes $\exp \{i k x\}$ and $\exp \{-i k x\}$ where $x$ is the distance to one of the vertices attached to the bond/lead. For all bonds/leads, the coefficients of the linear combination are determined by boundary conditions specified in terms of $V$ matrices $\Gamma^{(\alpha)}$ of dimension $V$ defined for each vertex $\alpha$. The matrix $\Gamma^{(\alpha)}$ expresses the outgoing amplitudes on the lead and on each of the $V-1$ bonds attached to vertex $\alpha$ (written symbolically as $\mathcal{O}$ ) in terms of the incoming amplitudes on the same or any other bond or the lead attached to $\alpha$ (written symbolically as $\mathcal{I}$ ) so that $\mathcal{O}=\Gamma^{(\alpha)} \mathcal{I}$. With $\beta, \gamma \neq \alpha$ the unitary and symmetric matrix $\Gamma^{(\alpha)}$ has the form

$$
\Gamma^{(\alpha)}=\left(\begin{array}{ll}
\rho^{(\alpha)} & \tau_{\gamma}^{(\alpha)} \\
\tau_{\beta}^{(\alpha)} & \sigma_{\beta \gamma}^{(\alpha)}
\end{array}\right) .
$$

Here $\rho^{(\alpha)}$ describes backscattering on lead $\alpha, \tau_{\beta}^{(\alpha)}$ describes scattering from bond $(\alpha \beta)$ to lead $\alpha$ or vice versa, and $\sigma_{\beta \gamma}^{(\alpha)}$ describes scattering from bond $(\alpha \beta)$ to bond $(\alpha \gamma)$ or vice versa. In general, the matrix $\sigma^{(\alpha)}$ is symmetric and subunitary. For a vertex $\alpha$ without lead the first row and column of $\Gamma^{(\alpha)}$ are lacking, we have $\Gamma^{(\alpha)}=\sigma^{(\alpha)}$, and $\sigma^{(\alpha)}$ is symmetric and unitary.

Given a single incident wave in channel $\alpha$ only, these boundary conditions completely define the total wave 
function. The amplitude of the outgoing wave in channel $\beta$ is the element $S_{\alpha \beta}(k)$ of the symmetric and unitary scattering matrix. To write $S_{\alpha \beta}(k)$ explicitly, we define the symmetric subunitary block-diagonal matrix $\Sigma$ of dimension $2 B=V(V-1)$. Each of the $V$ diagonal blocks of dimension $(V-1)$ carries one of the matrices $\sigma^{(\alpha)}$, $\alpha=1, \ldots, V$. All other elements of $\Sigma$ vanish. This defines the "vertex representation" $\Sigma^{(V)}$ of $\Sigma$. The "bond representation" $\Sigma^{(B)}$ is obtained by a reordering of rows and columns. We assign to every bond $(\alpha \beta)$ the direction $d=+(d=-)$ if $\alpha>\beta(\alpha<\beta$, respectively). We arrange the $B$ bonds with positive (negative) direction in lexicographical order and label them consecutively with a running index $b=1, \ldots, B$. Then every directed bond is uniquely defined by $(b, d)$. The total number of directed bonds is $2 B$. The map $\sigma_{\beta \gamma}^{(\alpha)} \rightarrow \sigma_{(\alpha \beta),(\alpha \gamma)}$ defines the bond representation of $\sigma^{(\alpha)}$ and, thus, the bond representation $\Sigma^{(B)}$ of $\Sigma$. The map $\tau_{\beta}^{(\alpha)} \rightarrow \tau_{(\alpha \beta)}$ similarly defines the bond representation of the vector $\mathcal{T}$. In bond representation we define the diagonal matrix $\exp \{-i k \mathcal{L}\}$ with elements $\delta_{b b^{\prime}} \delta_{d d^{\prime}} \exp \left\{-i k L_{b}\right\}$. Diagonal elements in locations that differ only in the sign of $d$ are pairwise equal. Written somewhat symbolically the $S$-matrix is

$$
S_{\alpha \beta}(k)=\delta_{\alpha \beta} \rho^{(\alpha)}+\left(\mathcal{T} \mathcal{W}^{-1} \mathcal{T}\right)_{\alpha \beta}
$$

where $\mathcal{W}=\exp \{-i k \mathcal{L}\}-\Sigma^{(B)}$. Expanding $\mathcal{W}^{-1}$ in powers of $\Sigma^{(B)}$ we obtain a simple physical interpretation of Eq. (2). The term containing the $n$th power of $\Sigma^{(B)}$ is the sum of all semiclassical trajectories that connect the vertices $\alpha$ and $\beta$ via passage through $(n+1)$ bonds. Each of the traversed bonds $(b d)$ yields the factor $\exp \left\{i k L_{b}\right\}$.

Averages. The average over $k$ (indicated by angular brackets) is taken over a $k$-interval that is larger than the minimum difference between any two $L_{b}$ 's. Because of the incommensurability of the $L_{b}$, that average is equivalent [5] to a phase average: For any function $F$ we have $\left\langle F\left[\exp \left\{i k L_{b}\right\}\right]\right\rangle=(1 /(2 \pi)) \int_{0}^{2 \pi} \mathrm{d} \phi_{b} F\left[\exp \left\{i \phi_{b}\right\}\right]$. Then Eq. (2) implies $\left\langle S_{\alpha \beta}\right\rangle=\delta_{\alpha \beta} \rho^{(\alpha)} 13$ and can, thus, be read as $S=\langle S\rangle+S^{\mathrm{fl}}$ where the fluctuating part is $S^{\mathrm{fl}}=\mathcal{T} \mathcal{W}^{-1} \mathcal{T}$. It also follows that the average of the product of any number of $S$-matrix elements is equal to the product of the averages (as in RMT).

Supersymmetry and saddle-point approximation. The $S$-matrix correlation function $(P, Q)$ is defined as the average of a product of $P$ elements of $S^{\mathrm{fl}}$ with arguments $k+\kappa_{p}, p=1, \ldots, P$ and $Q$ elements of $S^{\mathrm{f} *}$ with arguments $k-\tilde{\kappa}_{q}, q=1, \ldots, Q$. Without loss of generality we assume $P \geq Q \geq 1$. Since $S^{\mathrm{fl}}=\mathcal{T} \mathcal{W}^{-1} \mathcal{T}$, it suffices to work out

$$
\left\langle\prod_{p=1}^{P} \mathcal{W}_{b_{p} d_{p}, b_{p}^{\prime} d_{p}^{\prime}}^{-1}\left(k+\kappa_{p}\right) \prod_{q=1}^{Q}\left(\mathcal{W}_{b_{q} d_{q}, b_{q}^{\prime} d_{q}^{\prime}}^{-1}\left(k-\tilde{\kappa}_{q}\right)\right)^{*}\right\rangle .
$$

We generalize the approach of Refs. [5, 15]. Using supersymmetry [6, 16], the correlator (3) is written as the $(P+Q)$-fold derivative of the average of a generating function $G$ (a superintegral). The average over $k$ is calculated as a phase average over all $\phi_{b}$ with the help of the colour-flavour transformation [17] in its most general form (for $P \neq Q$ ). Integrating out the original integration variables gives

$$
\langle G\rangle=\int \mathrm{d}(\tilde{Z}, Z) \exp \{-\mathcal{A}(\tilde{Z}, Z)\}
$$

where $\mathcal{A}(\tilde{Z}, Z)$ is the action

$$
\begin{gathered}
\mathcal{A}(\tilde{Z}, Z)=-\mathrm{S} \operatorname{Tr} \ln (1-Z \tilde{Z})+\frac{1}{2} \mathrm{~S} \operatorname{Tr} \ln \left(1-Z z Z^{\tau} z\right) \\
+\frac{1}{2} \mathrm{~S} \operatorname{Tr} \ln \left(1-\mathcal{B}_{+}^{-1} \tilde{Z}^{\tau} z\left(\mathcal{B}_{-}^{\dagger}\right)^{-1} \tilde{Z} z\right)
\end{gathered}
$$

Here STr is the supertrace. All matrices are defined in bond and in retarded-advanced representation. In the retarded (advanced) sector, the matrix dimension is $8 B P$ ( $8 B Q$, respectively), a factor 4 arising from supersymmetry. The matrix $Z(\tilde{Z})$ fills the upper (lower) nondiagonal block of the retarded-advanced sector, respectively, and is rectangular for $P>Q$. Both $Z$ and $\tilde{Z}$ are diagonal in bond space. The matrices $Z$ and $Z^{\tau}$ are related as in Ref. [5]. The matrix $z$ is diagonal with diagonal elements given by $\exp \left\{i \kappa_{p} L_{b} / 2\right\}, p=1, \ldots, P$ in the retarded sector and by $\exp \left\{i \tilde{\kappa}_{q} L_{b} / 2\right\}, q=1, \ldots, Q$ in the advanced sector. The matrix $\mathcal{B}_{+}\left(\mathcal{B}_{-}\right)$is only defined for the retarded (the advanced) sector, respectively. $\mathcal{B}_{+}^{-1}$ is block-diagonal with regard to the index $p=1, \ldots, P$ and in each block given by $\Sigma_{b d, b^{\prime} d^{\prime}}^{(B)}+\sigma_{3}^{s} A^{(p)}$, and correspondingly for $\mathcal{B}_{-}^{-1}$. Here $\sigma_{3}^{s}$ is the third Pauli matrix in superspace, and $A^{(p)}$ denotes the source term needed to generate by differentiation of $G$ the matrix element $\mathcal{W}_{b_{p} d_{p}, b_{p}^{\prime} d_{p}^{\prime}}^{-1}$. The integration measure in Eq. (44) is the flat Berezinian. Up to this point our results are exact.

We calculate $\langle G\rangle$ using the saddle-point approximation, putting $z=1$ and $A^{(j)}=0$ for all $j$. As in Ref. [5], variation of the resulting action with respect to $Z$ and $\tilde{Z}$ yields the saddle-point equation $(1-Z \tilde{Z})^{-1} Z=$ $\left(1-\Sigma_{+} Z \Sigma_{-}^{*} \tilde{Z}\right)^{-1} \Sigma_{+} Z \Sigma_{-}^{*}$. Here $\Sigma_{+}$is block-diagonal and in each block labelled $p=1, \ldots, P$ given by $\Sigma^{(B)}$, and correspondingly for $\Sigma_{-}$. The saddle-point equation holds if $Z \Sigma_{-}^{*}=\Sigma_{+}^{*} Z$ and if $\Sigma^{(B)} \Sigma^{(B) *}=1$. We write $\Sigma^{(V)} \Sigma^{(V) *}=1+\delta^{(V)}, \Sigma^{(B)} \Sigma^{(B) *}=1+\delta^{(B)}$. As done in RMT, we first suppress $\delta^{(V)}$ and $\delta^{(B)}$ (both of which are caused by coupling to the channels). We then work out the ensuing corrections to the saddle-point solution exactly in Eq. (8) below. To satisfy $Z \Sigma_{-}^{*}=\Sigma_{+}^{*} Z$ we follow Ref. [5] and write the universal saddle-point solution $Y$ as $\delta_{b b^{\prime}} \delta_{d d^{\prime}} Y_{p t s, q t^{\prime} s^{\prime}}$, and correspondingly for $\tilde{Y}$.

Corrections to the saddle-point action are due to deviations from $z=1$, and from $\Sigma^{(B)} \Sigma^{(B) *}=1$. Concerning the former, we expand [5] $z$ and the action $\mathcal{A}$ around the saddle-point value up to first order in $\kappa_{p}$ and $\tilde{\kappa}_{q}$. With $\left\langle d_{\mathrm{R}}\right\rangle=(1 / \pi) \sum_{b} L_{b}$ the average level density [5], we obtain in the exponent of Eq. (4) the "symmetry-breaking 
term"

$$
S Y=i \pi\left\langle d_{\mathrm{R}}\right\rangle\left(\operatorname{STr}_{p s t} \kappa \frac{1}{1-Y \tilde{Y}}+\operatorname{STr}_{q s t} \tilde{\kappa} \frac{1}{1-\tilde{Y} Y}\right) .
$$

With $s, t$ labelling the superindices, the trace is only over the subspaces indicated, the matrix $\kappa$ is $\delta_{s s^{\prime}} \delta_{t t^{\prime}} \delta_{p p^{\prime}} \kappa_{p}$, and correspondingly for $\tilde{\kappa}$. Deviations from $\Sigma^{(B)} \Sigma^{(B) *}=$ 1 are calculated by putting $z=1$ and dropping the source terms. We use Eq. (11), suppress the index $\alpha$, and take $\rho$ to be real (as in RMT, that suppresses all elastic scattering phase shifts). Since $\Gamma$ is unitary and symmetric it can be unitarily transformed into

$$
\left(\begin{array}{ccc}
\rho & \exp \left\{-i \phi_{1}\right\} T^{1 / 2} & 0 \\
\exp \left\{-i \phi_{1}\right\} T^{1 / 2} & -\rho \exp \left\{-2 i \phi_{1}\right\} & 0 \\
0 & 0 & \delta_{\mu \nu} \exp \left\{i \phi_{\mu}\right\}
\end{array}\right)
$$

Here $T=1-\rho^{2}$ is the transmission coefficient, the indices $\mu, \nu$ run from 2 to $V-1$, and the phases $\phi_{1}, \phi_{\mu}$ are real and arbitrary. Eq. (7) shows that $\sigma \sigma^{*}$ differs from the unit matrix only in the first diagonal element which is $1-T$. Using that for all channels $\alpha$ we obtain in the exponent of Eq. (4) the "channel-coupling term"

$$
C H=-\frac{1}{2} \sum_{\alpha=1}^{V} \mathrm{STr}_{p s t} \ln \left(1+T^{(\alpha)} \frac{Y \tilde{Y}}{1-Y \tilde{Y}}\right) .
$$

Actually the sum extends only over the $\Lambda \ll V$ vertices coupled to a lead. Collecting everything we find

$$
\langle G\rangle=\int \mathrm{d}(Y, \tilde{Y})(\ldots) \exp \{S Y+C H\} .
$$

The term in big round brackets contains the source terms.

Massive Modes. In Eq. (9) we have neglected the massive modes because our interest in the present paper is focused on generating universal results for chaotic scattering without using the framework of RMT. Massive modes were investigated in Refs. [5, 15] for closed graphs. For the statistics of eigenfunctions it was found [15] that sequences of graphs with monotonically increasing $V$ are quantum ergodic (i.e., the massive modes do not contribute) if the spectrum of eigenvalues of the analogue of our matrix $\left|\Sigma_{b d, b^{\prime} d^{\prime}}^{(B)}\right|^{2}$ asymptotically $(V \rightarrow \infty)$ possesses a gap separating it from zero. Wave-function statistics is known [18] to be important for $S$-matrix fluctuations. Therefore, we conjecture that for $\Lambda \ll V$ ( $\Lambda$ fixed and $V \rightarrow \infty)$ that criterion applies in the present case to the spectrum of our $\left|\Sigma_{b d, b^{\prime} d^{\prime}}^{(B)}\right|^{2}$. A proof would require a detailed investigation.

Two-point Function. For the correlation function $(1,1)$ the matrices $Y$ and $\tilde{Y}$ are both square matrices of dimension four, and it is straightforward to work out the source terms in Eq. (9). Lack of space does not permit us to present any details. Suffice it to say that using the transformations $t_{12}=Y(1-Y \tilde{Y})^{-1 / 2}, t_{21}=\tilde{Y}(1-Y \tilde{Y})^{-1 / 2}$, writing $\left\langle S_{\alpha \alpha}\right\rangle$ for $\rho^{(\alpha)}$ and $T^{(\alpha)}=1-\left|\left\langle S_{\alpha \alpha}\right\rangle\right|^{2}$, and replacing the wave-number arguments of $S$ by energies, the resulting expressions for $\left\langle S_{\alpha \beta}^{\mathrm{fl}}(k+\kappa) S_{\gamma \delta}^{\mathrm{f} *}(k-\kappa)\right\rangle$ become formally identical to the corresponding terms in Eq. (7.23) of Ref. [6] for all values of the number $\Lambda=1, \ldots, V$ of channels. For the terms $S Y$ and $C H$ in Eqs. (6) and (8) that can be checked directly. In the source terms, the phase $\phi_{1}$ cancels out. These facts establish the equivalence of the two-point functions of RMT and of chaotic scattering on quantum graphs.

Ericson Regime. That regime is defined by the condition $\sum_{\alpha} T^{(\alpha)} \gg 1$. The cross section for chaotic scattering is expected to display Ericson fluctuations [10, 11]. Numerical simulations [13] have confirmed that expectation. Eq. (9) allows us to determine the leading terms in an asymptotic expansion in inverse powers of $\sum_{\alpha} T^{(\alpha)}$ of all $(P, Q)$-correlation functions and, thus, the complete distribution of $S$-matrix elements in the Ericson regime. The asymptotic terms are obtained [19] by keeping in Eq. (9) only terms of lowest order in $Y, \tilde{Y}$. For $S Y+C H$ we obtain

$$
-\frac{1}{2} \sum_{p q}\left(\sum_{\alpha} T^{(\alpha)}-2 i \pi\left\langle d_{\mathrm{R}}\right\rangle\left(\kappa_{p}+\tilde{\kappa}_{q}\right)\right) \operatorname{STr}_{s t}\left(Y_{p q} \tilde{Y}_{q p}\right) .
$$

For the two-point function, the calculation [19] yields

$$
\left\langle S_{\alpha \beta}^{\mathrm{f}}(k+\kappa) S_{\gamma \delta}^{\mathrm{fl} *}(k-\tilde{\kappa})\right\rangle=\frac{\left(\delta_{\alpha \gamma} \delta_{\beta \delta}+\delta_{\alpha \delta} \delta_{\beta \gamma}\right) T^{(\alpha)} T^{(\gamma)}}{\sum_{\tau} T^{(\tau)}-2 i \pi\left\langle d_{\mathrm{R}}\right\rangle(\kappa+\tilde{\kappa})} .
$$

With $T^{(\alpha)}=1-\left|\left\langle S_{\alpha \alpha}\right\rangle\right|^{2}$ and the replacement of wave numbers by energies, this is exactly the expression obtained for RMT in Refs. [8, 20]. For the general $(P, Q)$ correlation function we need to find the leading-order contribution to the source terms. We expand the last term in Eq. (5) with $\tilde{Z}^{\tau} \rightarrow Y$ and $\tilde{Z} \rightarrow \tilde{Y}$, retaining only terms linear in $Y$ and $\tilde{Y}$. (Only these are of the form $\sum_{p q} Y_{p q} \tilde{Y}_{q p}$ which, according to Eq. (10), gives the leading-order contribution). We need $P$ source terms from $\mathcal{B}_{+}$. Expanding the exponential we keep the term $\left(1 /\left(2^{P} P !\right)\right)\left[\sum_{p q} \operatorname{STr}_{s t}\left(\left(\mathcal{B}_{+}^{-1}\right)_{p} Y_{p q}\left(\mathcal{B}_{+}^{\dagger-1}\right)_{q} \tilde{Y}_{q p}\right)\right]^{P}$. No two source terms in $\mathcal{B}_{+}^{-1}$ may have the same labels. That gives $\left(1 / 2^{P}\right) \prod_{p}\left[\sum_{q} \operatorname{STr}_{s t}\left(\left(\sigma_{3}^{s} A^{(p)} Y_{p q}\left(\mathcal{B}_{+}^{\dagger-1}\right)_{q} \tilde{Y}_{q p}\right)\right]\right.$. Since all source terms in $\mathcal{B}_{-}^{\dagger-1}$ must also be different, the sum over $q$ goes for $P=Q$ over all permutations of $q=1, \ldots, Q$. Equivalently we may keep all $q$ fixed and sum over all permutations of $p=1, \ldots, P$. For $P>Q$ there are $P-Q$ source terms $A^{(p)}$ in $\mathcal{B}_{+}^{\dagger-1}$ that do not have a counterpart in $\mathcal{B}_{-}^{\dagger-1}$. For these $\mathcal{B}_{-}^{\dagger-1}$ is replaced by $\Sigma$. Each of the resulting supertraces corresponds to one of the factors in the flat integration measure $\mathrm{d}(Y, \tilde{Y})=\prod_{p q} \mathrm{~d}\left(Y_{p q}, \tilde{Y}_{q p}\right)$. Therefore and because of Eq. (10), each superintegral factorizes into $P Q$ terms, each factor characterized by the pair $(p, q)$ of indices. In factors that do not carry any source terms the superintegration gives unity. The integration over those supertraces which carry both factors $A^{(p)}$ and $A^{(q)}$ yields the asymptotic form (11) of the average of a pair of $S$-matrix elements. For the $P-Q$ unpaired source terms $A^{(p)}$ the superintegration gives a non-vanishing contribution for $\alpha_{p}=\beta_{p}$ only. The result- 
ing factor is

$$
\mathcal{F}_{\alpha_{p}}\left(\kappa_{p}\right)=-\sum_{q=1}^{Q} \frac{T^{\left(\alpha_{p}\right)}\left\langle S_{\alpha_{p} \alpha_{p}}\right\rangle}{\sum_{\gamma} T^{(\gamma)}-2 i \pi\left(\kappa_{p}+\tilde{\kappa}_{q}\right)\left\langle d_{\mathrm{R}}\right\rangle}
$$

where again the phase $\phi_{1}$ in Eq. (7) cancels out. The sum over $q$ arises because in the advanced block, the matrix $\mathcal{B}_{-}^{\dagger-1}$ carries the same entry $\Sigma$ in every block labelled $q$. We suppress all arguments $k$ for brevity. That gives

$$
\begin{aligned}
& \left\langle\prod_{p=1}^{P} S_{\alpha_{p} \beta_{p}}^{\mathrm{fl}}\left(\kappa_{p}\right) \prod_{q=1}^{Q} S_{\alpha_{q}^{\prime} \beta_{q}^{\prime}}^{\mathrm{fH} *}\left(-\tilde{\kappa}_{q}\right)\right\rangle=\sum_{\text {sel }} \prod_{j=1}^{P-Q} \mathcal{F}_{\alpha_{p_{j}}}\left(\kappa_{p_{j}}\right) \\
& \times \sum_{\operatorname{perm}} \prod_{q=1}^{Q}\left\langle S_{\bar{\alpha}_{q} \bar{\beta}_{q}}^{\mathrm{f}}\left(\bar{\kappa}_{q}\right) S_{\alpha_{q}^{\prime} \beta_{q}^{\prime}}^{\mathrm{f} *}\left(-\tilde{\kappa}_{q}\right)\right\rangle .
\end{aligned}
$$

The sum with index "sel" goes over all $\left(\begin{array}{c}P \\ P-Q\end{array}\right)$ possibilities to select $(P-Q)$ matrix elements $S^{\mathrm{fl}}$ from the first factor on the left-hand side. These give rise to the first product which vanishes unless all selected elements are diagonal. The remaining $Q$ elements $S^{\mathrm{fl}}$, symbolically written as $S_{\bar{\alpha}_{j} \bar{\beta}_{j}}^{\mathrm{f}}\left(\bar{\kappa}_{j}\right)$ with $j=1, \ldots, Q$, appear as first factors in the angular brackets on the right-hand side. The sum with index "perm" extends over all permutations of these elements. Each of the terms in angular brackets on the right-hand side is equal to the asymptotic form (11) of the two-point function.

Eq. (13) gives the asymptotic form of all $S$-matrix correlation functions $(P, Q)$ and, thus, the complete distribution of the $k$-dependent scattering matrix in the Ericson regime. For the $(2,2)$ correlation function it agrees with the result of Ref. [8]. If $\left\langle S_{\alpha \alpha}\right\rangle=0$ for all $\alpha$ (strong absorption in all channels), the factors $\mathcal{F}$ all vanish, the correlation functions $(P, Q)$ vanish for $P \neq Q$, and for $P=Q$ have the form characteristic of a Gaussian random process. In particular, all elements of $S$ have a
Gaussian distribution centered at zero, and cross-section fluctuations have the form predicted in Refs. [10, 11]. If $\left\langle S_{\alpha \alpha}\right\rangle \neq 0$ in some channel $\alpha$, that fact and the unitarity constraint $\left|S_{\alpha \alpha}\right| \leq 1$ distort the Gaussian distribution of $S_{\alpha \alpha}$. This is the cause of the occurrence of the factor $\mathcal{F}_{\alpha}$ (Eq. (12)). The factor shows that the distortion is biggest for $\left|\left\langle S_{\alpha \alpha}\right\rangle\right|=1 / \sqrt{2}$. For cross-section correlation functions, interest is focussed on the $(2,1)$ correlation function. It was first noted in Refs. [9] that this function differs from zero (the result given there agrees aympotically with our Eq. (13)). Implications of that fact for cross-section fluctuations have been discussed in Ref. [21].

Conclusions. For chaotic scattering on quantum graphs we have derived formal analytical expressions for all $(P, Q)$ correlation functions of the $S$-matrix. These were used to show that the $(1,1)$ correlation function is identical to the one obtained from RMT, and to calculate for all $(P, Q)$ explicit expressions in the Ericson regime. The latter agree with RMT results as far as these are known and yield the complete $S$-matrix distribution function in that regime. (It may perhaps be possible to obtain from Eq. (9) explicit expressions also for $(2,1)$ and $(2,2)$.) We conjecture that our results apply asymptotically for sequences of quantum graphs that when closed are quantum ergodic.

Two facts suggest that our results are universal, i.e., hold for quantum-chaotic scattering in general: The agreement of our results with those of RMT, and the agreement of the two-point function for closed graphs [5] with that of general closed chaotic systems [4]. Therefore, we conjecture that quantum-chaotic scattering and the RMT approach to scattering are completely equivalent.

ZP acknowledges support by the Czech Ministry of Education under Project MSM 0021620859. The authors are grateful for valuable comments to A. Altland, S. Gnutzmann, J. Kvasil, P. Cejnar, and U. Smilansky.
[1] F. J. Dyson, J. Math. Phys. 3, 1199 (1962).

[2] O. Bohigas, M.-J. Giannoni, and C. Schmit, Phys. Rev. Lett. 52, 1 (1984).

[3] S. Müller, S. Heusler, P. Braun, F. Haake, and A. Altland, Phys. Rev. Lett. 93, 014103 (2004) and Phys. Rev. E 72, 046207 (2005).

[4] S. Heusler, S. Müller, A. Altland, P. Braun, and F. Haake, Phys. Rev. Lett. 98, 044103 (2007) and New J. Phys. 11, 103025 (2009).

[5] S. Gnutzmann and A. Altland, Phys. Rev. Lett. 93, 194101 (2004) and Phys. Rev. E 72, 056215 (2005).

[6] J. J. M. Verbaarschot, H. A. Weidenmüller, and M. R. Zirnbauer, Phys. Rep. 129, 367 (1985).

[7] Y. V. Fyodorov, D. V. Savin, and H. J. Sommers, J. Phys. A 38, 10731 (2005).

[8] D. Agassi, H. A. Weidenmüller, and G. Mantzouranis, Phys. Rep. 22, 145 (1975).

[9] E. D. Davis and D. Boose, Phys. Lett. B 211, 379 (1988) and Z. Phys. A 332, 427 (1989).

[10] T. Ericson, Phys. Rev. Lett. 5, 430 (1960) and Ann. Phys. (N.Y.) 23, 390 (1963).

[11] D. M. Brink and R. O. Stephen, Phys. Lett. 5, 77 (1963).

[12] T. Kottos and U. Smilansky, Phys. Rev. Lett. 85, 968 (2000).

[13] T. Kottos and U. Smilansky, J. Phys. A: Math. Gen. 36, 3501 (2003).

[14] T. Kottos and H. Schanz, Physica E 9, 523 (2001) and Waves in Random Media 14, 591 (2004).

[15] S. Gnutzmann, J. P. Keating, and F. Piotet, Phys. Rev. Lett. 101, 264102 (2008) and Ann. Phys. (N.Y.) 325, 2595 (2010).

[16] K. B. Efetov, Adv. Phys. 32, 53 (1983).

[17] M. R. Zirnbauer, J. Phys. A: Math. Gen. 29, 7113 (1996).

[18] G. E. Mitchell, A. Richter, and H. A. Weidenmüller, Rev. Mod. Phys. 82, 2845 (2010).

[19] J. J. M. Verbaarschot, Ann. Phys. (N.Y.) 168, 368 
(1986).

[21] B. Dietz et al., Phys. Lett. B 685, 263 (2010).

[20] H. A. Weidenmüller, Ann. Phys. (N.Y.) 158, 120 (1985). 